\title{
Transcatheter Closure of Complex Post-Myocardial Infarction Left Ventricular Pseudoaneurysm and Unique Post-Traumatic Right Ventricular Pseudoaneurysm
}

\author{
Liang Tang, ${ }^{1}$ MD, Jian-jun Tang, ${ }^{1}$ MD, Xin-qun Hu, ${ }^{1}$ MD, Zhen-fei Fang, ${ }^{1}$ MD, Zhao-wei Zhu, ${ }^{1}$ MD, \\ Ya-qin Chen, ${ }^{1}$ MD, Yan-shu Zhao, ${ }^{1}$ MD, Ran Peng, ${ }^{1}$ MD and Sheng-hua Zhou, ${ }^{1}$ MD
}

\begin{abstract}
Summary
Ventricular pseudoaneurysm (PSA) is a rare, yet life-threatening complication of myocardial infarction, cardiac surgery, and transcatheter valve replacement. Although conventional surgery is the preferred treatment strategy, transcatheter closure has emerged as an effective alternative in selected candidates. In this report, we describe successful transcatheter closure of two unique cases of ventricular pseudoaneurysm (PSA): first, a complex post-myocardial infarction left ventricular PSA (LVPSA) with multi-communications, and second, a case of post-traumatic right ventricular PSA (RVPSA) following blunt chest injury caused by domestic violence.
\end{abstract}

(Int Heart J 2019; 60: 998-1002)

Key words: Blunt chest injury

$\mathrm{V}$ entricular pseudoaneurysm (PSA) results from a rupture of the ventricular free wall that is contained by the adherent pericardium, thrombus or scar tissue. ${ }^{1)}$ It is a rare, yet life-threatening complication that may occur following acute myocardial infarction (AMI), cardiac surgery, trauma, or transcatheter valve replacement. ${ }^{2-6)}$ If left untreated, ventricular PSA carries a $30-45 \%$ risk of rupture within the first year, significantly increasing the risk of sudden cardiac death. Thus, aggressive treatment is recommended in all cases regardless of whether the patient is symptomatic., ${ }^{3,4)}$ Surgical repair remains the mainstay therapy of ventricular PSA; however, surgical repair is associated with a high morbidity and mortality, especially when performed in an emergency setting and in patients with multiple comorbidities. ${ }^{2)}$ Recent reports suggest that percutaneous closure is an appropriate treatment alternative in high-risk or inoperable surgical candidates with ventricular PSAs, ${ }^{1,3-8)}$ but the experience with this procedure is still limited. In this report, we describe two cases of ventricular PSA: first, a complex postmyocardial infarction left ventricular PSA (LVPSA) with multi-communications, and second, a case of posttraumatic right ventricular PSA (RVPSA) following blunt chest injury caused by domestic violence. Both cases were successfully managed percutaneously with "off-label" use of ventricular septal occluders.

\section{Case Report}

Case 1: A 79-year-old man with a history of coronary artery bypass grafting 6 years earlier was admitted with pro- gressively worsening shortness of breath for 10 days after an anterior AMI. He was also known to have had a prior stoke. Cardiac examination revealed a systolic-diastolic murmur at the apex. Transthoracic echocardiography (TTE) revealed a huge saccular PSA originating from the apex, with two defects and pulsatile flow from the apex into the PSA cavity (Figure 1A). Cardiac magnetic resonance imaging (MRI) showed thin, dyskinetic anterolateral, apex akinesia and a large LVPSA at the apex with a well-defined neck (Figure 1B). There was concern for impending rupture. Given his advanced age, multiple comorbidities, and prior sternotomy, the patient was deemed extremely high risk for surgical repair by the heart team and was thus referred for consideration of percutaneous management. The informed consent process for the planned percutaneous procedure included discussion of the "off-label" use of the occluder devices, and the potential risks of thrombus dislodgment, device embolization, and procedure-related rupture of the PSA. The patient was taken to the Hybrid OR suite and monitored anesthesia care was used with deep conscious sedation. Right femoral arterial access was obtained and heparin was administered to maintain an activated clotting time $($ ACT $)>250$ seconds. A left ventriculogram evidenced a huge, pulsatile LVPSA (Figure 1C). Taking into consideration that a delivery sheath advanced from the femoral artery would make it difficult to reach the LVPSA as the delivery sheath was too short due to the tortuosity in the aorta, we decided to close it via a right brachial access. The PSA was entered with a $5 \mathrm{~F}$ multipurpose catheter and a 0.035 inch hydrophilic glidewire (Terumo Medical Corp, Japan).

From the ${ }^{1}$ Department of Cardiology, The Second Xiangya Hospital of Central South University, Changsha, China.

Address for correspondence: Sheng-hua Zhou, MD, Department of Cardiology, The Second Xiangya Hospital of Central South University, No.139, Middle Ren-min Road, Changsha, Hunan, 410011, China. E-mail: zhoushenghua@csu.edu.cn

Received for publication September 26, 2018. Revised and accepted December 21, 2018

Released in advance online on J-STAGE June 28, 2019.

doi: 10.1536/ihj.18-577

All rights reserved by the International Heart Journal Association. 

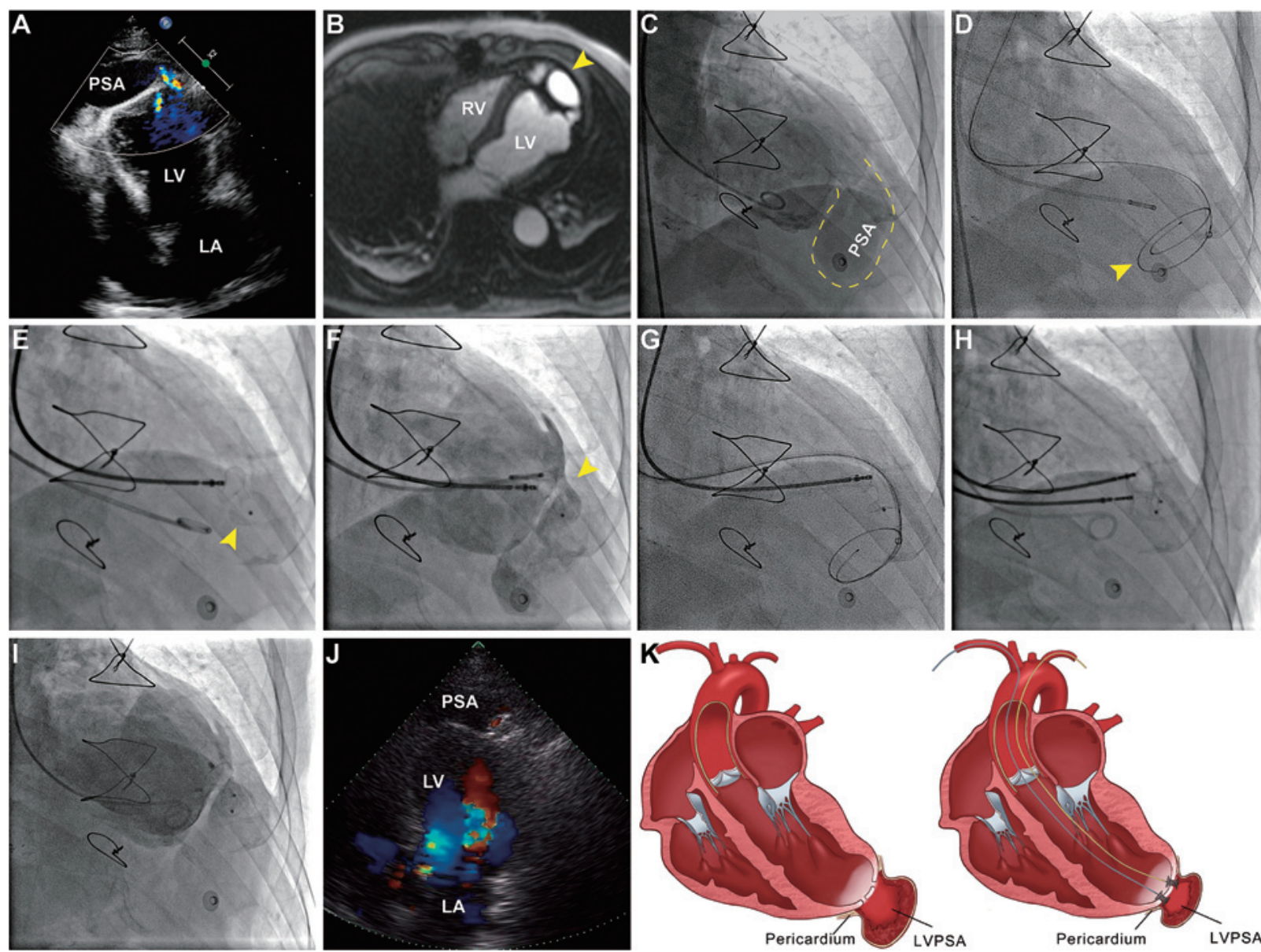

Figure 1. Transcatheter one-stage closure of left ventricular pseudoaneurysm (LVPSA) with multi-communications using multiple occluders. A: Transthoracic echocardiogram (TTE) revealed a huge saccular LVPSA with two communications. B: Magnetic resonance imaging confirmed the presence of an LVPSA (arrowhead). C: Left ventriculogram revealed a huge PSA. D: Delivery sheath was advanced over a 0.025-inch coiled floppy tip guidewire into the cavity (arrowhead). E: A $10 \mathrm{~mm}$ muscular ventricular septal defect occluder (MVSDO, arrowhead) was deployed across the first defect. F: Repeat ventriculogram clearly delineating the other communication (arrowhead) to the PSA. G and H: The second communication was successfully treated with a $12 \mathrm{~mm}$ MVSDO. I: Final ventriculogram showing well-placed occluders without significant shunt. J: TTE performed one month later confirmed no significant residual shunt. K: Schematic diagram of transcatheter closure of LVPSA using two MVSDOs via bilateral brachial artery approach. LA indicates left atrium; LV, left ventricular; PSA, pseudoaneurysm; and RV, right ventricle.

To avoid wire perforation, a 0.025 -inch coiled floppy tip guidewire (Toray Industries, Japan) was then exchanged into the PSA and over which a 7F delivery sheath was advanced into its cavity (Figure 1D). Under echocardiographic and fluoroscopic guidance, a $10 \mathrm{~mm}$ muscular ventricular septal defect occluder (MVSDO, Lifetech Ltd, Shenzhen, China) was successfully deployed across the defect (Figure 1E). A repeat ventriculogram clearly delineated the other access connected to the PSA, with a welldefined neck measuring $8 \mathrm{~mm}$ (Figure $1 \mathrm{~F}$ ). Via the left brachial access, this communication was successfully treated in a similar fashion with a $12 \mathrm{~mm}$ MVSDO (Figures $1 \mathrm{G}, 1 \mathrm{H})$. The final ventriculogram confirmed that these two occluders were well-positioned with mild residual leak expected to resolve once the device was thrombosed and endothelialized (Figure 1I). The patient made a slow but uneventful recovery and was discharged one week later. Repeat TTE performed one-month postprocedure showed well-positioned devices without a sig- nificant residual shunt and no intraventricular thrombus (Figure 1J).

Case 2: A 50-year-old, otherwise healthy woman was referred to our institution for evaluation of oppressive retrosternal chest discomfort which she had been experiencing for 10 months. Her symptoms occurred following an episode of severe domestic violence; the patient reported that her husband struck her chest and back with his fists and knee 10 months previously. On admission, cardiac examination revealed a grade $2 / 6$ systolic murmur at her lower left sternal border. Chest x-rays showed old 4th and 5 th rib fractures of the left hemithorax. TTE revealed a large saccular PSA arising from the right ventricular (RV) apex with to-and-fro flow in the communicating tract (Figure 2A). Cardiac MRI confirmed the presence of a right ventricular PSA (RVPSA), with a neck measuring 5 $\mathrm{mm}$ in its maximum diameter (Figure $2 \mathrm{~B}$ ). Coronary angiography showed normal coronary arteries. Based on these findings and her past medical history, we speculated that 

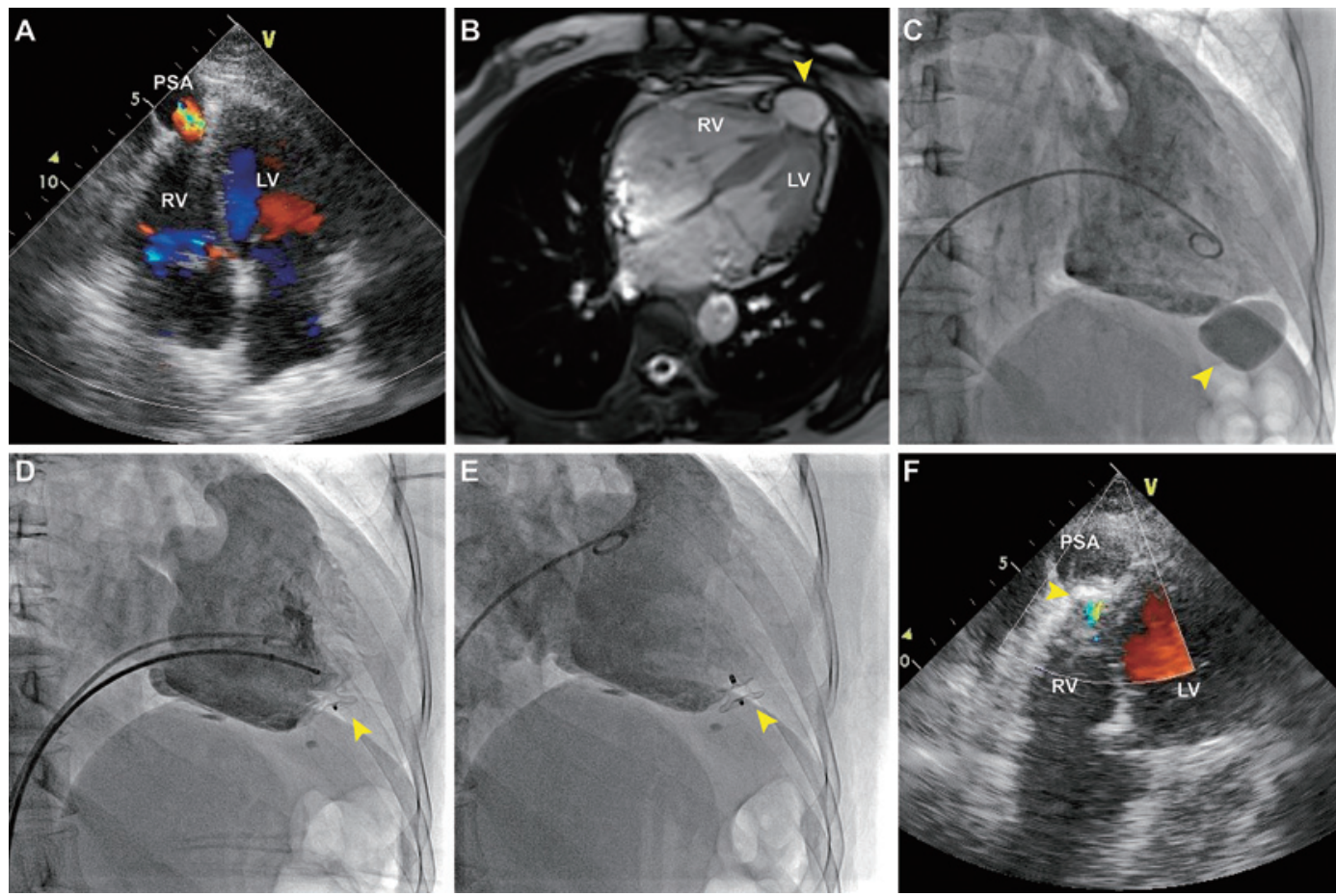

Figure 2. Transcatheter closure of post-traumatic right ventricular pseudoaneurysm. A: Transthoracic echocardiogram (TTE) revealing a pseudoaneurysm (PSA) arising from the right ventricle (RV) apex. B: The PSA was further characterized by cardiac magnetic resonance imaging (arrowhead). C: Right ventriculogram revealing the large PSA (arrowhead) with a focal neck. D and E: Repeat ventriculogram shows occlusion of the PSA with use of an $8 \mathrm{~mm}$ peri-membranous ventricular septal occluder (arrowhead). F: Repeat TTE performed 6-months post procedure revealed well-positioned closure device (arrowhead) with no flow into the PSA. LV indicates left ventricular.

the RVPSA might have been caused by the blunt chest trauma from the domestic abuse. Considering the future risk of PSA rupture, surgical intervention was recommended. However, the patient declined surgical repair and preferred transcatheter therapy. Therefore, the decision was made for percutaneous closure of the RVPSA. Written informed consent was obtained from the patient after detailed explanation of the procedure including information about therapeutic alternatives, procedural risks, and "off-label" use of closure devices. The procedure was performed under light sedation, via a femoral venous access. Intravenous heparin was administered during the procedure. A right ventriculogram further characterized the large PSA at the RV apex (Figure 2C). Under fluoroscopy and TTE guidance, the PSA was selectively engaged with a 5F Judkins right catheter and a 0.035-inch angled hydrophilic glidewire. The glidewire was then exchanged for a 0.035-inch Extra-Support Amplatz wire (Boston Scientific, USA) over which a $6 \mathrm{~F}$ delivery sheath was successfully advanced into the aneurysm. An $8 \mathrm{~mm}$ perimembranous VSD occluder (Lifetech Ltd, Shenzhen, China) was then deployed across the defect. Repeat ventriculography showed no significant flow into the PSA sac (Figures 2D, 2E). The patient was discharged the following day and she was symptom-free at 6-month follow-up. Repeat TTE performed 6-months post-procedure confirmed a well-seated closure device with no residual flow (Figure 2F).

\section{Discussion}

Surgical closure remains the mainstay of therapy and is urgently recommended when ventricular PSA complicates AMI early on (within the first 2-3 months), because onset of rupture is unpredictable. ${ }^{2)}$ Recently, the "offlabel" use of a variety of Amplatzer occluders has enabled the transcatheter therapy of a handful of high-risk surgical patients with ventricular PSAs. ${ }^{3-8)}$ However, the experience of transcatheter closure of ventricular PSA is limited, due to the rarity of its occurrence. Thus far, very limited data exists regarding the patient selection of transcatheter closure of ventricular PSAs. Most of the treatment decisions were based on data from the previously published isolated case reports or small case series. According to previous reports, all patients with ventricular PSAs were discussed in a multidisciplinary manner by cardiovascular surgeons and cardiologists and patients who were treated percutaneously were deemed high-risk surgical candidates. ${ }^{4-11)}$ More importantly, specific favorable anatomical conditions are critical to obtain satisfactory results. For transcatheter closure of ventricular PSAs, sealing to eliminate residual leaks as well as robust anchoring of the occluder device are mandatory. Therefore, this procedure is predominantly performed in patients with a well-defined neck which can secure the occluder device within the orifice portion of the PSA. ${ }^{1,4-11)}$ For patients with wide-mouthed large PSAs without an ideal landing zone, transcatheter closure may 
not be feasible due to inadequate occluder device anchoring which may lead to a high risk of device embolization. Additionally, Kumar, et al. . $^{3}$ proposed that when planning transcatheter closure of LV outflow tract PSAs, external compression involving the left main and the left anterior descending arteries by the occluder devices should be taken into consideration. In contrast, structures in the atrioventricular groove (circumflex and the coronary sinus) are of concern in transcatheter closure of posterobasal ventricular PSAs. It is also worth noting that a cerebrovascular event due to embolization of thrombus originated from the PSA sac has been described as a potential complication during the procedure. ${ }^{9)}$ Therefore, in patients with large ventricular PSAs filled by massive thrombus which possess a high risk of thrombotic embolization, transcatheter closure should be performed with caution. It is reasonable to postpone the procedure until after full anticoagulation therapy.

Multimodality imaging and an appropriate approach to treatment proved essential to the transcatheter closure procedure with an excellent outcome. The procedural strategies need to be tailored individually according to the anatomic characteristics of PSAs: the size and location of the PSA orifice; width and length of the neck; shape of the PSA cavity, and structures adjacent to the PSA. Since there is no dedicated device designed for this application, the percutaneous closure relies on "off-label" use of a variety of Amplatzer occluder devices. Among them, the muscular VSD occluder has a 7-mm waist that best accommodates to the LV myocardium tissue, and hence, it is frequently used for LVPSA closure. ${ }^{12)}$ Certainly, the selection of a device for a given case primarily depends on the anatomical features of the PSA that are mentioned above. For a PSA with a long neck (as in the present Case 1), devices that have a lengthier waist, such as an Amplatzer muscular VSD occluder, Duct occluder, or Vascular Plug might be suitable devices. ${ }^{1}$ For a PSA with a short neck (the thickness of the orifice site was less than that of the neighboring myocardium), as in the present patient 2, an Amplatzer septal occluder or peri-membranous VSD occluder might be an appropriate device, as the thickness of the waist of the occluder is only $4 \mathrm{~mm}$. This was considered essential for stability and to achieve complete closure. In addition, the application of a combination of coils to embolize the PSA cavity with an Amplatzer occluder device to close the neck of the PSA has also been described. ${ }^{10,13)}$ The routes that are commonly used for percutaneous closure include retrograde transaortic, transapical, and anterograde transseptal routes, and direct chest wall puncture. ${ }^{4-8)}$ For the present case 1 , the brachial artery approach was chosen (the schematic diagram is given in Figure $1 \mathrm{~K}$ ), as the length of the delivery system was not sufficient for a femoral artery route. An alternative would have been an antegrade transseptal route, but the aneurysm with two orifices would not favor this method.

While LVPSAs are an uncommon condition, RVPSAs are even more rarely described and no report has specified the incidence of RVPSA after blunt chest injury. The possible mechanisms of traumatic ventricular PSA following blunt chest injury include a cardiac contusion, or rapid deceleration that provokes excessive shear forces on the ventricular free wall and intramyocardial dissecting hematoma. ${ }^{14-16)}$ To date, very few cases of transcatheter closure of RVPSA have been described. Agnoletti, et al. ${ }^{17}$ reported the successful percutaneous closure of an RVPSA using an Amplatzer septal occluder in a 25-year-old man with complex congenital heart disease who had undergone 3 surgical operations. Alkhouli, et al. ${ }^{18)}$ recently described a case of an acquired RVPSA following pericardiocentesis, which was successfully treated with percutaneous closure. In their case, the aneurysmal cavity was approached by direct percutaneous needle chest wall puncture, and the RVPSA was excluded by the combination of an Amplatzer Vascular Plug II device and thrombin injection into the sac to expedite the clot formation. To the best of our knowledge, the 2 present cases might represent the first description of successful transcatheter one-stage closure of post-myocardial infarction LVPSA with multicommunications via a bilateral brachial artery approach, as well as post-traumatic RVPSA following blunt chest injury due to domestic violence.

\section{Conclusion}

The present cases demonstrate that transcatheter onestage closure of complex post-myocardial infarction LVPSA with multi-communications as well as posttraumatic RVPSA following blunt chest injury is a feasible treatment option. For those in whom conventional surgery cannot be performed or patients where a less invasive option is desired, catheter-based treatment can be undertaken.

\section{Disclosures}

Conflicts of interest: The authors have no conflicts of interest to disclose.

\section{References}

1. Dudiy Y, Jelnin V, Einhorn BN, Kronzon I, Cohen HA, Ruiz CE. Percutaneous closure of left ventricular pseudoaneurysm. Circ Cardiovasc Interv 2011; 4: 322-6.

2. Eren E, Bozbuga N, Toker ME, et al. Surgical treatment of postinfarction left ventricular pseudoaneurysm: a two-decade experience. Tex Heart Inst J 2007; 34: 47-51.

3. Kumar PV, Alli O, Bjarnason H, Hagler DJ, Sundt TM, Rihal CS. Percutaneous therapeutic approaches to closure of cardiac pseudoaneurysms. Catheter Cardiovasc Interv 2012; 80: 687-99.

4. Okuyama K, Chakravarty T, Makkar RR. Percutaneous transapical pseudoaneurysm closure following transcatheter aortic valve replacement. Catheter Cardiovasc Interv 2018; 91: 159-64.

5. Feldman T, Pearson P, Smart SS. Percutaneous closure of post TAVR LV apical pseudoaneurysm. Catheter Cardiovasc Interv 2016; 88: 479-85.

6. Salaun E, Aldebert P, Jaussaud N, et al. Early endocarditis and delayed left ventricular pseudoaneurysm complicating a transapical transcatheter mitral valve-in-valve implantation: percutaneous closure under local anesthesia and echocardiographic guidance. Circ Cardiovasc Interv 2016; 9: e003886.

7. Singh A, Kliger C, Ruiz CE. Novel approach for the percutaneous treatment of left ventricular pseudoaneurysms. Catheter Cardiovasc Interv 2015; 85: 1092-6.

8. Matsumoto T, Okuyama K, Cheng W, Makkar RR, Kar S. 
Transseptal closure of left ventricular pseudoaneurysm posttransapical transcatheter aortic valve replacement. JACC Cardiovasc Interv 2014; 7: e177-8.

9. Narayan RL, Vaishnava P, Goldman ME, et al. Percutaneous closure of left ventricular pseudoaneurysm. Ann Thorac Surg 2012; 94: e123-5.

10. Acharya D, Nagaraj H, Misra VK. Transcatheter closure of left ventricular pseudoaneurysm. J Invasive Cardiol 2012; 24: e1114.

11. Karimi A, Beaver TM, Fudge JC Jr. Percutaneous transfemoral closure of a pseudoaneurysm at the left ventricular apical access site for transcatheter aortic valve implantation. J Invasive Cardiol 2015; 27: e27-9.

12. Mendiz O, Fava C, Cerda M, Lev G, Caponi G, Valdivieso L. Percutaneous repair of left ventricular pseudoaneurysm after transcatheter aortic valve replacement. Cardiovasc Revasc Med 2017; 18: 30-1.

13. Yudi MB, Love B, Nadir A, Kini A, Sharma SK. Percutaneous closure of left ventricular pseudoaneursym with septal occluder device and coils: A multimodality imaging approach. JACC Cardiovasc Interv 2017; 10: e159-61.

14. Chen F, Wei S, Xiong L, Liu F. Post-traumatic left ventricular outflow tract pseudoaneurysm. Ann Thorac Surg 2014; 97: 3112.

15. Bozlar U, Yurtsever I, Ugurel MS, Ors F, Nural MS, Tasar M. Multidetector computed tomography in post-traumatic ventricular pseudoaneurysm. Clin Cardiol 2009; 32: e72-4.

16. Tang L, Tang JJ, Fang ZF, Hu XQ, Shen XQ, Zhou SH. Severe mechanical hemolysis after transcatheter closure of a traumatic ventricular septal defect using the Amplatzer atrial septal occluder. Int Heart J 2016; 57: 519-21.

17. Agnoletti G, Iserin L, Rizzo E, Mousseaux E. Percutaneous closure of a false aneurysm of the right ventricle in a congenital heart disease patient. Circulation 2007; 115: e400-2.

18. Alkhouli M, Waits B, Chaturvedi A, Ling FS. Percutaneous closure of right ventricular pseudoaneurysm. JACC Cardiovasc Interv 2015; 8: e147-8. 